\title{
The topographic bias in gravimetric geoid determination revisited
}

DOI: https://doi.org/10.1515/jogs-2019-0007

Received February 1, 2019; accepted April 26, 2019

\begin{abstract}
The topographic potential bias at geoid level is the error of the analytically continued geopotential from or above the Earth's surface to the geoid. We show that the topographic potential can be expressed as the sum of two Bouguer shell components, where the density distribution of one is spherical symmetric and the other is harmonic at any point along the normal to a sphere through the computation point. As a harmonic potential does not affect the bias, the resulting topographic bias is that of the first component, i.e. the spherical symmetric Bouguer shell. This implies that the so-called terrain potential is not likely to contribute significantly to the bias. We present three examples of the geoid bias for different topographic density distributions.
\end{abstract}

Keywords: density distribution, geoid, geopotential, terrain, topographic bias

\section{Introduction}

As the determination of the Earth's potential by Stokes' formula requires no masses outside the sphere of integration, the effect of the topographic masses need special treatment. In the remove-compute-restore technique (e.g., Sjöberg and Bagherbandi, 2017, Sect. 6.2.1) it means that the effect is first removed from the gravity observations (the direct topographic effect) prior to Stokes integration and then added back as a potential correction (the indirect topographic effect) to the preliminary computed geoid height.

In contrast, the KTH method (e.g. Sjöberg 2003, Sjöberg and Bagherbandi 2017, Sect. 6.2.2) first determines a preliminary geoid height by Stokes' formula using surface gravity anomalies, which result is corrected for the error caused by its harmonic/analytical continuation (AC) through the topography to the geoid. The resulting geoid height is biased whenever the geoid is located inside the topography, and the bias, which is caused only by the fact

*Corresponding Author: Lars E. Sjöberg: Royal Institute of Technology (KTH) Stockholm, Sweden, E-Mail: Isjo@kth.se that the topographic potential is not harmonic along the vertical between the computation point and its foot point at the geoid, we call the topographic bias (of the geoid height). This bias can be treated as the sum of that for a Bouguer shell through the computation point and that for the remaining topography (the terrain effect). Sjöberg (2007, 2008 and 2009a, b) claimed that (from a practical view) the total bias is that of the Bouguer shell, although the final proof that there is no contribution from the terrain in the near zone is still debated. See also Wang (1990).

Here we will reconsider the bias using a slightly different approach. However, we will not discuss the bias in the AC when using an external type spherical harmonic expansion of the geopotential; see Sjöberg and Bagherbandi (2017, Sect. 5.2.6.)

\section{The topographic potential and its bias in analytical continuation}

The topographic potential at an arbitrary point $P$ can be determined by the Newton integral

$V_{P}^{T}=\iint_{\sigma} \int_{R}^{r_{s}} \mu \frac{r^{2}}{l_{p}} d r d \sigma$, where $l_{P}=\sqrt{r_{P}^{2}+r^{2}-2 r_{p} r \cos \psi}$

For $r_{P}=R$ one arrives at the topographic potential at sea/geoid level:

$V_{0}^{T}=\iint_{\sigma} \int_{R}^{r_{s}} \mu \frac{r^{2}}{l_{0}} d r d \sigma$, where $l_{0}=\sqrt{R^{2}+r^{2}-2 R \cos \psi}$.

Here $\sigma$ is the unit sphere, $\mu$ is gravitational constant times density of mass, $\psi$ is the geocentric angle between computation and integration points, $R, r_{P}, r$ and $r_{s}$ are the radii at sea level (or geoid level, approximated by a sphere), computation and integration points and topographic surface above or below the computation point, respectively.

The AC either upward or downward of the topographic potential, denoted $\left(V_{P}^{T}\right)^{\star}$, can be performed by the Taylor 
series of $V_{P}^{T}$ at $P$ along the vertical (Wang 1990; HofmannWellenhof and Moritz 2005, Sect.8.6; Sjöberg 2009a):

$$
\left(V_{P}^{T}\right)^{*}=\sum_{k=0}^{\infty} \frac{\left(-h_{P}\right)^{k}}{k !} \frac{\partial^{k} V_{P}^{T}}{\partial r_{P}^{k}}
$$

where $h_{P}=r_{P}-R$. If the AC is carried out in free space, downward or upward, implying that the potential is harmonic and all its radial derivatives exist between $P$ and the foot point of the geoid, then $\left(V_{P}^{T}\right)^{\star}=V_{0}^{T}$, otherwise it is biased. Hence, the bias of the (topographic) potential is defined by

$$
\operatorname{bias}\left(V_{P}^{T}\right)=\left(V_{P}^{T}\right)^{\star}-V_{0}^{T} \text {. }
$$

The bias is frequently divided into those of the potentials of a Bouguer shell $\left(V_{P}^{B}\right)$ and the remaining topographic potential, the terrain potential $\left(V_{P}^{\text {terr }}\right)$, defined as follows:

$$
\operatorname{bias}\left(V_{P}^{T}\right)=\operatorname{bias}\left(V_{P}^{B}\right)+\operatorname{bias}\left(V_{P}^{\text {terr }}\right),
$$

where

$$
V_{P}^{B}=\iint_{\sigma} \int_{R}^{r_{0}} \mu \frac{r^{2}}{l_{p}} d r d \sigma
$$

and

$$
V_{P}^{t e r r}=\iint_{\sigma} \int_{r_{Q}}^{r_{s}} \mu \frac{r^{2}}{l_{p}} d r d \sigma .
$$

Here $r_{Q}$ is the surface radius along the vertical through $P$. (Note that $P$ is arbitrarily located on or outside the topography.)

\section{The bias of the Bouguer shell}

In case of a Bouguer plate potential of thickness $H$ and constant density $\mu_{0}$ Wang (1990) showed that the bias becomes $2 \pi \mu_{0} H^{2}$, and similarly for a spherical Bouguer shell potential Sjöberg (2007) came to the bias of $2 \pi \mu_{0} H^{2}(1+$ $\left.\frac{2 H}{3 R}\right)$, where $H$ is the thickness and $R$ the inner radius of the shell.

In Sect. 3.1 we will consider the topographic density as spherically symmetric, while in Sect. 3.2 it is generalized to an arbitrary distribution.

\subsection{The bias for a spherical symmetric density distribution}

Here we consider the bias of a spherical Bouguer shell between the spheres of radii $R$ and $r_{Q}>R$, having a spherical symmetric layered density distribution $\mu(r)$. The potential of the shell becomes

$$
\begin{aligned}
V_{P}^{B} & =2 \pi \int_{R}^{r_{O}} \mu(r) r^{2} \int_{1}^{-1} \frac{d t}{l_{P}} d r \\
& =2 \pi \int_{R}^{r_{O}} \mu(r) r^{2} \frac{r_{P}+r-\left|r_{P}-r\right|}{r_{P} r} d r,
\end{aligned}
$$

where the surface element of Eq. (1) was written

$$
d \sigma=-\sin \psi d \psi d \alpha=d t d \alpha, t=\cos \psi
$$

and $\alpha$ is azimuth.

Equation (6a) can be decomposed into an external potential

$$
V_{P}^{B, e}=4 \pi \int_{R}^{r_{Q}} \mu(r) \frac{r^{2}}{r_{P}} d r, \text { if } r_{P} \geq r_{Q}
$$

and an internal potential (at the geoid or below):

$$
V_{P}^{B, i}=4 \pi \int_{R}^{r_{Q}} \mu(r) r d r, \text { if } r_{P} \leq R,
$$

and, finally, if $R<r_{P}<r_{Q}$ :

$$
V_{P}^{B, e+i}=4 \pi \int_{R}^{r_{P}} \mu(r) \frac{r^{2}}{r_{P}} d r+4 \pi \int_{r_{P}}^{r_{Q}} \mu(r) r d r .
$$

By taking the difference of the potentials in Eqs. (7a) and (7c) at any point inside the shell, one finds the bias for the external Bouguer shell potential:

$$
\operatorname{bias}\left(V_{P}^{B}\right)^{\star}=4 \pi \int_{r_{P}}^{r_{Q}} \mu(r)\left(\frac{r^{2}}{r_{P}}-r\right) d r ; R \leq r_{P} \leq r_{Q},
$$

which vanishes at the surface with $r_{P}=r_{Q}$ and becomes

$$
\operatorname{bias}\left(V_{P}^{B}\right)^{\star}=\left(V_{P}^{B}\right)^{\star}-V_{0}^{B}=4 \pi \int_{R}^{r_{Q}} \mu(r)\left(\frac{r^{2}}{R}-r\right) d r
$$

at the geoid (with radius $R$ ).

In particular, for $\mu=\mu(r)=$ constant and $r_{Q}=R+H_{P}$, the bias at geoid level becomes

$$
\operatorname{bias}\left(V_{P}^{B}\right)=4 \pi \mu\left(\frac{r^{3}}{3 R}-\frac{r^{2}}{2}\right)_{r=R}^{r=R+H_{P}}=2 \pi \mu\left(H_{P}^{2}+\frac{2 H_{P}^{3}}{3 R}\right) .
$$

See also Sjöberg (2007). 


\subsection{The bias for an arbitrary density distribution}

Let us now decompose the arbitrary density $\mu$ (which may vary in 3D) into a radial symmetric distribution $\mu_{A}(r)$ and the residual component $v_{A}=\mu-\mu_{A}(r)$. Here the sub index $A$ means a selected point on the sphere such that $\mu_{A}(r)=\mu\left(r, \Omega_{A}\right)$, where $\Omega=(\varphi, \lambda)=$ (latitude, longitude), implying that $v_{A}(r)=0$ at any point along the vertical through $A$. Then the potential of the Bouguer shell between spheres of radii $R$ and $r_{Q}>R$ can be written (cf. Eq. 1):

$$
\begin{aligned}
V_{P}^{B} & =\iint_{\sigma} \int_{R}^{r_{Q}}\left(\mu_{A}(r)+v_{A}\right) \frac{r^{2}}{l_{P}} d r d \sigma \\
& =\iint_{\sigma} \int_{R}^{r_{Q}} \mu_{A}(r) \frac{r^{2}}{l_{P}} d r d \sigma+\iint_{\sigma} \int_{R}^{r_{O}} v_{A} \frac{r^{2}}{l_{P}} d r d \sigma \\
& =V_{P}^{B, 1}+V_{P}^{B, 2} .
\end{aligned}
$$

It follows directly from Sect. 3.1 that the bias of $V_{P}^{B, 1}$ is the same as that in Eq. (9) with $\mu=\mu_{A}$.

Finding the bias of $V_{P}^{B, 2}$ is more complicated. If one applies the Laplace operator to each component of Eq. (10a), one obtains, term by term from Laplace's and Poisson's equations:

$$
0=0+0
$$

if $P$ is located on or outside the shell, and

$$
-4 \pi \mu=-4 \pi \mu_{A}-4 \pi v_{A},
$$

if $P$ is located inside the shell.

In the special case that $P$ is located inside the shell along the vertical through $A$, Eq. (10c) becomes

$$
-4 \pi \mu=-4 \pi \mu_{A},
$$

implying that $v_{A}=0$ and $\Delta v_{A}=0$ at any point along this vertical. Hence, it is tempting to suggest that $V_{P}^{B, 2}$ is harmonic along the vertical so that this potential does not contribute to the topographic bias in AC. However, as stated by Kellog (1953, p. 211), a potential is harmonic at a point $P$ only "if its second derivatives exist and are continuous and satisfy Laplace's equation throughout some neighborhood of that point”. That is, satisfying the Laplacian at the point is not a sufficient condition for being harmonic, but in the present case a thin (but finite) hole along the vertical from the geoid to the surface would be sufficient. Hence, one may only state that $V_{P}^{B, 2}$ is approximately unbiased, or

$$
\operatorname{bias}\left(V_{P}^{B, 2}\right) \approx 0 \text {. }
$$

F. Sansó (private communication) presented a simple counter example of the potential of a spherical shell with a laterally variable density. Although the example shows that the downward continued potential is biased even along the normal where the density vanishes, we found that this error is only about $11 \mu \mathrm{m}$ at the peak of Mt. Everest (see Appendix).

Hence, from the discussion in Sect. 3.1 and above, Eq. (10a) leads to the following practical bias of the AC of the external Bouguer shell potential at the geoid:

$$
\operatorname{bias}\left(V_{P}^{B}\right)^{\star}=\left(V_{P}^{B}\right)^{\star}-V_{0}^{B}=4 \pi \int_{R}^{r_{0}} \mu_{A}(r)\left(\frac{r^{2}}{R}-r\right) d r,
$$

i.e. the bias is practically the same as that for the spherical symmetric layered shell.

\section{The bias of the terrain potential}

The mass of the terrain is the topographic mass not included in the Bouguer shell. A general formula for the terrain potential was given in Eq. (5c). Wang (1990) found the following estimate of its bias for a constant topographic density of the terrain:

$$
V_{\text {bias }}^{\text {terr }} \approx \frac{3 \mu}{8} h_{P}^{4} \iint_{A} \frac{h-h_{s}}{x^{2}++y^{2}+h^{2}} d x d y,
$$

where $A$ is the horizontal- or $(x, y)$-plane. However, this estimate is the result of a series expansion truncated at power 2, and the truncation error is not included. Sjöberg (2009a) found that the terrain correction/bias vanishes or is limited to the integration over a small portion of the terrain around the computation point. Here we will be more precise in estimating this potential component.

\subsection{New results for the terrain bias}

The terrain potential of Eq. (5c) can be decomposed into

$$
V_{P}^{\text {terr }}=V_{P}^{\text {terr }+}+V_{P}^{\text {terr- }},
$$

where

$$
V_{P}^{t e r r+}=\iint_{\sigma} \int_{r_{Q}}^{r_{s+}} \mu \frac{r^{2}}{l_{p}} d r d \sigma \geq 0
$$

and

$$
V_{P}^{t e r r-}=\iint_{\sigma} \int_{r_{Q}}^{r_{s-}} \mu \frac{r^{2}}{l_{p}} d r d \sigma \leq 0
$$


where $r_{s+}$ and $r_{s-}$ are the surface radii for terrain masses above and below the sphere of radius $r_{Q}$.

It is obvious that $V_{P}^{\text {terr- }}$ could have been included as part of the Bouguer shell potential $V_{P}^{B, 2}$, which is harmonic and therefore without bias. Also $V_{P}^{\text {terr }}$ is harmonic for $r_{P}<r_{Q}$, suggesting that it does not contribute to the bias in the AC to the geoid. Next we present a proof.

If $r_{P}$ is located in the interval $R \leq r_{P} \leq r_{Q}$, then $V_{P}^{\text {terr }+}$ can be expressed as a convergent inner type Laplace series

$$
V_{P}^{t e r r+}=\sum_{n=0}^{\infty} \frac{r_{P}^{n}}{r_{Q}^{n}} V_{n}^{+}
$$

where

$$
V_{n}^{+}=\frac{2 n+1}{4 \pi} \iint_{\sigma} \int_{r_{Q}}^{r_{Q}^{n}} \mu \frac{r^{2}}{r^{n-1}} d r P_{n}(\cos \psi) d \sigma .
$$

Here $P_{n}(\cos \psi)$ is the $n$-th Legendre's polynomial.

In particular, for $r_{P}=R$

$$
V_{0}^{t e r r+}=\sum_{n=0}^{\infty} \frac{R^{n}}{r_{Q}^{n+1}} V_{n}^{+},
$$

which is the terrain potential at the geoid.

It remains to show that $\mathrm{AC}$ of the surface terrain potential, given by Eq. (16a), equals Eq. (17). Hence, by inserting Eq. (16a) into Eq. (3) one obtains

$$
\left(V_{P}^{\text {terr }+}\right)^{*}=\sum_{k=0}^{\infty} \frac{\left(-h_{P}\right)^{k}}{k !} \sum_{n=0}^{\infty} \frac{n(n-1) \ldots(n-k+1) r_{P}^{n-k}}{r_{Q}^{n+1}} V_{n}^{+}
$$

or

$$
\left(V_{P}^{\text {terr+ }}\right)^{\star}=\sum_{n=0}^{\infty} \frac{V_{n}^{+}}{r_{Q}^{n+1}} I_{n}\left(r_{P}, h_{P}\right)
$$

where

$$
I_{n}\left(r_{P}, h_{P}\right)=\sum_{k=0}^{n} \frac{n(n-1) \ldots(n-k+1)}{k !} r_{P}^{n-k}\left(-h_{P}\right)^{k},
$$

which is the binomial series of $\left(r_{P}-h_{P}\right)^{n}$, i.e.

$$
I_{n}\left(r_{P}, h_{P}\right)=\sum_{k=0}^{n}\left(\begin{array}{c}
n \\
k
\end{array}\right) r_{P}^{n-k}\left(-h_{P}\right)^{k}=\left(r_{P}-h_{P}\right)^{n}=R^{n} .
$$

Therefore, by inserting Eq. (19c) into Eq. (19a) one arrives at Eq. (17), which shows that there is no contribution to the topographic bias from the terrain potential, $V_{P}^{\text {terr }}$. This result is a consequence of that the terrain potential is harmonic along the radius vector at the computation point $P$, implying that it is correctly continued to the geoid in the AC process.

\section{A refined approach}

In the following we will avoid the approximation of sea level to a sphere. Introducing the radii $R_{0}$ and $r_{B}$ of the enveloping/Brillouin and Bjerhammar spheres, respectively, with $R_{0}>\left(r_{s}\right)_{\max }$ and $r_{B} \leq\left(r_{g}\right)_{\min }$, where $r_{g}$ is the radius of the geoid, the topographic potential can be expressed:

$$
V_{P}^{T}=\iint_{\sigma} \int_{r_{B}}^{R_{0}} \mu \frac{r^{2}}{l_{p}} d r d \sigma
$$

which is identical to Eq. (1) as $\mu=0$ for $r_{B} \leq r<r_{g}$ and $r_{s}<r \leq R_{0}$. Inserting the substitution

$\mu=\mu_{A}(r)+v_{A}$ into Eq. (20), where $\mu_{A}(r)$ and $v_{A}$ were defined in Sect. 3.2, one obtains

$$
\begin{aligned}
V_{P}^{T}= & \iint_{\sigma} \int_{r_{B}}^{R_{0}} \mu_{A}(r) \frac{r^{2}}{l_{p}} d r d \sigma+\iint_{\sigma} \int_{r_{B}}^{R_{0}} v_{A} \frac{r^{2}}{l_{p}} d r d \sigma \\
& =V_{P}^{T, 1}+V_{P}^{T, 2} .
\end{aligned}
$$

$V_{P}^{T, 2}$ is harmonic for $r>r_{P}$ and for $r \leq r_{P}$ the bias is not significant, yielding

$$
\left(V_{P}^{T}\right)^{\star}=\left(V_{P}^{T, 1}\right)^{\star}=4 \pi \int_{r_{B}}^{R_{0}} \mu_{A}(r) \frac{r^{2}}{r_{g}} d r=4 \pi \int_{r_{g}}^{r_{0}} \mu_{A}(r) \frac{r^{2}}{r_{g}} d r,
$$

and the bias becomes

$$
\operatorname{bias}\left(V_{P}^{T}\right)^{*}=4 \pi \int_{r_{g}}^{r_{Q}} \mu_{A}(r)\left(\frac{r^{2}}{r_{g}}-r\right) d r .
$$

In case that the geoid is located above the Earth's surface, it holds that $\mu_{A}(r)=0$ in Eq. (23), and the bias correctly vanishes.

\section{Examples}

Example 1: If the topographic density (denoted $\mu_{0}$ ) is constant, Eq. (23) yields

$$
\operatorname{bias}\left(V_{P}^{T}\right)^{\star}=2 \pi \mu_{0}\left(H^{2}+\frac{2 H^{3}}{3 r_{g}}\right),
$$

where $H$ is the orthometric height at $P$.

Example 2: Assume that the density is distributed into two layers, such that it is constantly $\mu_{0}$ from the surface down to radius $r_{1}$ and then constantly $\mu_{1}$ down to the geoid at 
radius $r_{g}$. Then Eq. (23) yields the bias

$$
\operatorname{bias}\left(V_{P}^{T}\right)^{\star}=4 \pi \mu_{1} \int_{r_{g}}^{r_{1}}\left(\frac{r^{2}}{r_{g}}-r\right) d r+4 \pi \mu_{0} \int_{r_{1}}^{r_{0}}\left(\frac{r^{2}}{r_{g}}-r\right) d r \text {. }
$$

Comparing with the bias for a constant topographic density in Eq. (24), there is here and additional bias of

$$
\operatorname{\Delta bias}\left(V_{P}^{T}\right)^{\star}=2 \pi \Delta \mu\left(H_{1}^{2}+\frac{2 H_{1}^{3}}{3 r_{g}}\right),
$$

where $\Delta \mu=\mu_{1}-\mu_{0}$ and $H_{1}=r_{1}-r_{g}$.

Example 3: Let the density be $\mu_{0}$ at the topographic surface and linearly changing to $\mu_{1}$ at the geoid. Then the density at radius $r, r_{g} \leq r \leq r_{Q}$, can be expressed:

$$
\mu(r)=\mu_{0}+\left(\mu_{1}-\mu_{0}\right) \frac{r_{Q}-r}{H}=\mu_{0} \frac{r-r_{g}}{H}+\mu_{1} \frac{r_{Q}-r}{H},
$$

so that in this case there is an additional bias at the geoid of

$$
\operatorname{\Delta bias}\left(V_{P}^{T}\right)^{\star}=2 \pi \Delta \mu\left(\frac{H^{2}}{3}+\frac{H^{3}}{6 r_{g}}\right) .
$$

Comparing with the bias of Example 1 (denoted bias $_{0}$ ), one can see that the relative additional bias becomes

$$
\frac{\Delta \text { bias }}{\text { bias }_{0}}=\frac{\Delta \mu}{\mu_{0}} \frac{1+H / 2 r_{g}}{3+2 H / r_{g}} \approx \frac{\Delta \mu}{3 \mu_{0}} .
$$

Hence, if the densities at the surface and geoid are 2.7 and $3.3 \mathrm{~g} / \mathrm{cm}^{3}$, the approximate relative additional bias is 7.4 $\%$, independent of topographic height.

\section{Concluding remarks}

We have shown that the potential of an arbitrary topographic density distribution can be decomposed into two Bouguer shell potentials, where one component has a spherical symmetric density distribution, and the other is practically harmonic at any point along the normal to the Brillouin sphere through the computation point. As the topographic bias is not affected by a harmonic potential, it is practically determined only by the first component. Compared to earlier investigations, this study refines the bias by not using a spherical approximation of the geoid radius, and it generalizes the result to an arbitrary density distribution.

The result implies that gravimetric geoid models can be carried out without the terrain correction, and the formal terrain correction determined in an arbitrarily thin (but finite) channel between the geoid and the surface along the vertical through the computation point, is likely not significant in real cases as shown by the example in Appenix. This can be performed as a two-step procedure: first the disturbing potential at the surface point is determined; second an AC procedure of this potential to the geoid yields a biased potential that needs a correction (which is actually the simple Bouguer plate correction; see Sjöberg 2015).

Note that the series for the AC of Eq. (3) is infinite. In this view, a practical obstacle could be that the rougher the terrain is around the computation point, the higher degree of truncation of the series is needed to fulfil a certain requirement of accuracy.

In Example 3 we showed that for a linearly increasing topographic density with depth from $2.7 \mathrm{~g} / \mathrm{cm}^{3}$ at the surface to $3.3 \mathrm{~g} / \mathrm{cm}^{3}$ at the geoid, there is an additional analytical continuation bias of $7 \%$ (independent of topographic height) vs. that for the constant topographic density $2.7 \mathrm{~g} / \mathrm{cm}^{3}$.

The method discussed here for analytical continuation of the topographic potential down to the geoid could be extended for the inversion of the gravity potential to any depth within the Earth, e.g. to the Moho.

Acknowledgement: I appreciate a constructive discussion with F. Sansó.

\section{References}

Hofmann-Wellenhof, B. and Moritz, H., 2005. Physical Geodesy, Springer Verlag, Vienna and New York

Kellog, O. D., 1953. Foundations of potential theory. Dover Publ., Inc., New York

Sjöberg, L. E., 2003. A computational scheme to model the geoid by the modified Stokes's formula without gravity reduction. J. Geod. 77,423-432

Sjöberg L. E., 2007. The topographic bias by analytical continuation in physical geodesy. J. Geod. 81, 345-350

Sjöberg, L. E., 2009a. The terrain correction in gravimetric geoid determination - is it needed? Geophys. J. Int. 176,14-18

Sjöberg, L.E., 2009b. On the topographic bias in geoid determination by the external gravity field, J. Geod. 83, 967-972.

Sjöberg, L. E., 2015. Rigorous geoid-from-quasigeoid corrections using gravity disturbances. J. Geod. Sci. 5, 115-118

Sjöberg, L. E. and Joud S., 2018. A numerical test of the topographic bias. J. Geod. Sci. 8: 14-17

Sjöberg, L. E. and Bagherbandi, M., 2017. Gravity inversion and integration, Springer Int. Publ. AG

Wang, Y. M., 1990. The effect of topography on the determination of the geoid using analytical downward continuation, Bull. Geod. 64, 231-246 


\section{A Appendix}

Example A1. (F. Sanso's counterexample: The AC for a spherical shell with a laterally variable density)

Let the density of a spherical shell be a function of the co-latitude $\theta$ :

$$
\mu(\theta)=\mu_{0}\left[1-Y_{10}(\theta)\right],
$$

where $Y_{10}=\sqrt{3} \cos \theta$ is the fully normalized first degree zonal spherical harmonic, implying that the density changes with co-latitude between $(1-\sqrt{3}) \mu_{0}$ and $(1+\sqrt{3}) \mu_{0}$, where $\mu_{0}$ is a constant.

Generally, the potential of the shell, bounded by the spheres of radii $R_{1}$ and $R$ with $R_{1}>R$, can be written

$$
V(P)=\iint_{\sigma} \mu \int_{R}^{R_{1}} \mu \frac{r^{2} d r}{l_{p}} d \sigma .
$$

In the exterior case (with $r_{P} \geq R_{1}$ ) this potential can be expanded to

$$
\begin{aligned}
V(P)= & \iint_{\sigma} \mu_{0}\left[1-Y_{10}(\Theta)\right] \\
& \int_{R}^{R} \sum_{n=0}^{1} \frac{r^{n+2} d r}{(2 n+1) r_{P}^{n+1}} \sum_{m=-n}^{n} Y_{n m}(\theta, \lambda) d \sigma Y_{n m}(P),
\end{aligned}
$$

or, after lateral followed by radial integration:

$$
\begin{aligned}
V(P) & =4 \pi \mu_{0} \int_{R}^{R_{1}}\left(\frac{r^{2}}{r_{P}}-\frac{r^{3}}{3 r_{P}^{2}} Y_{10}(P)\right) d r \\
& =\frac{4 \pi \mu_{0}}{3}\left[\frac{R_{1}^{3}-R^{3}}{r_{P}}-\frac{R_{1}^{4}-R^{4}}{4 r_{P}^{2}} Y_{10}\left(\theta_{P}\right)\right] .
\end{aligned}
$$

Similarly, the potential for $r_{P} \leq R$ after expanding $1 / l_{P}$ as an internal type series, becomes

$$
V(P)=\mu_{0} \int_{R}^{R_{1}} \sum_{n=0}^{\infty} \frac{r_{P}^{n}}{(2 n+1) r^{n-1}} Y_{n m} d r Y_{n m}(P),
$$

or

$$
V(P)=4 \pi \mu_{0}\left[\frac{R_{1}^{2}-R^{2}}{2}-\frac{r_{P}\left(R_{1}-R\right)}{3} Y_{10}\left(\theta_{P}\right)\right]
$$

We now limit the following discussion by focusing on the analytical continuation and its true value for $\theta_{P}=54 .^{\circ} 7356$, where $Y_{10}\left(\theta_{P}\right)=1$. Then, upon setting $R_{1}=R+H$, the AC of (A4) down to the radius $r_{P}=R$ yields,

$$
V^{\star}=4 \pi \mu_{0}\left[\frac{2 R H}{3}+\frac{H^{2}}{2}-\frac{H^{4}}{12 R^{2}}\right],
$$

and the true potential at radius $r_{P}=R$ (assumed to be at the geoid) follows from Eq. (A6) as:

$$
V^{\text {true }}(P)=4 \pi \mu_{0}\left(\frac{2 R H}{3}+\frac{H^{2}}{2}\right) .
$$

Hence, using Bruns' formula to get the topographic bias of the geoid height due to the terrain of becomes

$$
\operatorname{bias}(N)=\frac{V^{\star}-V^{\text {true }}}{y_{0}}=-\frac{\pi \mu_{0} H^{4}}{3 R^{2} y_{0}},
$$

where $y_{0}$ is normal gravity on the reference ellipsoid. Hence, formally there is a contribution of the terrain to the topographic bias. However, if the density of mass in $\mu_{0}$ is set to $2670 \mathrm{~kg} / \mathrm{m}^{3}$ and $R$ is $6371 \mathrm{~km}$, for the height of Mt. Everest $(8.848 \mathrm{~km})$ the bias becomes only $-3 \mu \mathrm{m}$, which is negligible.

If one studies the bias at an arbitrary point on the spherical shell with the density $\mu=\mu_{0}\left(1-Y_{10}\right)$, then the bias can be expressed:

$$
\operatorname{bias}(N)=\text { Post }+ \text { Dev, }
$$

where

$$
\text { Post }=\frac{2 \pi \mu_{0}}{y_{0}}\left(H^{2}+\frac{2 H^{3}}{3 R}\right)
$$

is the postulated bias, and

$$
\text { Dev }=-\frac{\pi \mu_{0} H^{4}}{3 y_{0} R^{2}} Y_{10}
$$

is the error of Post, which is insignificant at any point on the shell. 